\title{
SULLA DETERMINAZIONE DEGLI ELEMENTI INTRINSECI, FONDAMENTALI, DELLA SUPERFICIE TERRESTRE, MEDIANTE MISURE LOCALI.
}

\author{
Memoria di Adolfo Viterbi (Pavia).
}

Adunazaz del to novembre 1907 .

I. Le ricerche dirette a determinare la vera figura della superficie matematica terrestre (Geoide) hanno portato, come é ben noto, alla conclusione essere la forma di tale superficie sistematicamente diversa da quella dell'ellissoide di rivoluzione (ed anche da quella di un ellissoide a tre assi).

Tuttavia l'opportuna combinazione di considerazioni attinenti alla teoria meccanica della figura dei pianeti con le conclusioni dedotte dalle ricerche geodetiche, sperimentali, di varia indole, sino ad ora eseguite, portatono ad ammettere che gli elementi, dei quali si valgono i Geodeti per rappresentare le deviazioni del Geoide dalla forma ellissoidica e più particolarmente da un dato ellissoide di rivoluzione opportunamente determinato (ellissoide di BeSSEL) siano misurati da quantita sufficientemente piccole (rapporto alle dimensioni della terra) da potersi trascurare le loro potenze superiori alla prima e le quantiti di analogo ordine. Per conseguenza; cosa pure ben nota, la ricerca della vera forma del Geoide viene ordinariamente ricondotta alla determinazione dei predetti elementi, risguardati come quantiti piccole del $I^{0}$ ordine, assumendo sempre l'ellissoide di Besser, o, quanto meno, speciali ellissoidi di rivoluzione varianti dall'una all'altra regione, come superficie di riferimento alla quale si riducono e sulla quale si eseguiscono i calcoli inerenti alle varie operazioni geodetiche. Ed è superfluo ricordare come a tale intento sia informato l'indirizzo degli odierni studi di alta Geodesia.

Cionullameno sorse, come è ben naturale, l'idea di procedere anche ad uno studio più generale del Geoide, abbandonando, cioè, qualunque speciale ipotesi ed induzione, cercando, in ultima analisi, di dedurre i caratteri geometrici della superficie in parola semplicemente dai dati delle osservazioni. In tale indirizzo vanno segnalati i lavori del Christoffel ${ }^{\mathbf{x}}$ ), del Pizzetrit $^{2}$ ), del $\operatorname{Reina}^{3}$ ). Appunto la lettura di tali lavori, e

r) Ueber die Bestimmung der Gestalt einer krummen Oberfläche durch lokale Messungen auf derselben [Journal für die reine und angewandte Mathematik, t. LXIV (1865), pp. 193.209].

2) Contribuzione allo studio geometrico della superficie terrestre [Giornale della Società di Letture e Conversazioni scientifiche di Genova, vol. XVII (1887), fasc. di agosto-settembre, pp. 5-I7].

3) Sulla determinazione dei raggi di curvatura di una superficie per mezzo di misure locali sopra di essa [Rendiconti della R. Accademia dei Lincei, vol. II (1893), $2^{\circ}$ semestre, pp. 287-295].

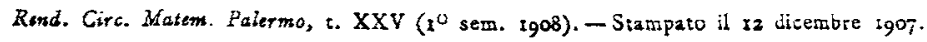


segnatamente di quello del prof. Pizzetri, mi suggerì l'idea di ricercare un sistenı completo ed omogeneo di formule, le quali da misure locali geodetiche ed astronomiche, opportunamente eseguite nei singoll punti della superficie terrestre, permettano di dedurre, in sostanza, le espressioni dei coefficienti delle due forme differenziali quadratiche fondamentali, relative a tale superficie. $E$ facilmente si perviene, come si vedrà, a formule, le quali, mediante convenienti quadrature, possono almeno teoricamente condurre anche alla determinazione dell'equazione del Geoide in termini finiti.

$E$ bensi vero che uno studio del Geoide, fondato sulle considerazioni che saranno qui svolte, non sarà ancora consentito dalle odierne risorse della Geodesia: tuttavia è presumibile che esso sard, in avvenire, reso possibile, mercè il continuo perfezionarsi dei metodi di osservazione proprî di tale scienza.

La trattazione stessa dell'argomento mi portó poi a stabilire alcuni risultati generali, di carattere matematico, che, sebbene non presentino vero interesse dal punto di vista geodetico, mi parve però opportuno rilevare.

Il punto di partenza della presente ricerca è costituito da alcune relazioni e formule stabilite dal prof. PizzetTi nel suo lavoro citato.

Ottengo poi in parte risultati già ottenuti, per altra via, rispettivamente dal CHRIStoffel e dal Reina nelle memorie citate, in parte altri risultati semplici ed elementari, dei quali non mi fu peró dato trovare cenno nei lavori di Geodesia venuti a mia conoscenza.

Ora, anche prima di procedere ad una esposizione generica del piano del lavoro, è bene, per maggior chiarezza, riportare alcune definizioni che hanno, per l'argomento qui trattato, speciale importanza ${ }^{4}$ ).

Presa pertanto a considerare la superficie terrestre, indichiamo con $z$ l'asse polare, con $\pi$ un piano fisso (scelto ad arbitrio) passante per questo asse.

Per un generico punto $P$ della superficie in parola, condotta la $P N$ normale alla superficie e la parallela $P M$ all'asse polare, si dirà al solito latitudine astronomica del punto $P$ il complemento dell'angolo $M P N$ che la direzione esterna della normale fa con la direzione Nord dell'asse polare e longitudine astronomica del punto stesso l'angolo che il piano $M P N$ fa col piano fisso $\pi$. Il piano $M P N$ dicesi piano del meridiano astronomico del punto $P$, e l'intersezione $P Q$ di esso col piano tangente, in $P$, alla superficie, si dirà linea cardinale Nord: a sua volta si dirà linea cardinale Est la retta $P E$ contenuta pure nel piano tangente considerato, e perpendicolare alla $P Q$. Saranno considerate, come direzioni positive sopra queste due rette, le direzioni che vanno verso Nord e verso Est rispettivamente.

Diremo al solito meridiano geoidico ogni linea luogo di punti di uguale longitudine astronomica, e parallelo geoidico ogni linea luogo di punti di uguale latitudine astronomica. Si dirà finalmente azimut astronomico di una linea uscente da $P$ e tracciata sulla

4) Le definizioni in parola sono tolte completamente dal citato lavoro del prof. PIzzetri ${ }^{2}$ ). 
superficie in parola l'angolo che la tangente alla linea stessa in $P$ forma con la direzione cardinale Nord.

All'asse polare $z$ associeremo poi altri due assi $x, y$ ortogonali ad esso e fra di loro, fissati in guisa che il piano $z x$ sia lo stesso piano fisso $\pi$, di cui sopra si disse. Disporremo in tal guisa di un sistema di assi cartesiani ortogonali $x, y, z$, al quale andranno riferiti i punti della superficie studiata.

In ció che segue presupporremo soltanto che siano sodisfatte alcune condizioni generali molto late, fatta eventualmente eccezione per punti isolati.

Tali condizioni si puó ammettere siano, per quanto riflette il Geoide, certo largamente sodisfatte e rientrano nella categoria di quelle che si pongono a base per la valiảità delle ordinarie formule di Geometria differenziale. A questo proposito devesi per altro ricordare che il Geoide va concepito come una superficie di livello, esteriore, senza di che non sarebbero sodisfatte talune delle condizioni che seguono. Le condizioni stesse sono:

$I^{\circ}$ Che la latitudine astronomica e la longitudine astronomica dei singoli punti di ciascuna linea della superficie in parola, come pure gli azimut astronomici delle tangenti di ogni linea (riferiti cioè alle linee cardinali Nord dei singoli punti) variino con continuitd lungo la linẹa ogni volta considerata e che, oltre ad essere funzioni continue della lunghezza d'arco della curva, ammettano, come tali, derivate determinate e finite.

$2^{\circ}$ Che le coordinate cartesiane, ortogonali $x, y, z$ dei punti del Geoide, nel sistema di assi assunto, considerate come funzioni delle coordinate geografiche (astronomiche) siano funzioni finite e continue ed ammettano anche derivate del primo e del secondo ordine almeno, determinate, finite e continue.

Valendomi pertanto di alcune delle proposizioni e formule dovute al Pizzetri, incomincio con lo stabilire relazioni le quali permettono di dedurre, per ciascun punto del Geoide, dai risultati delle ordinarie operazioni di Astronomia geodetica (determinazioni di coordinate astronomiche di punti e di azimut di direzioni) opportunamente eseguite:

$I^{\circ}$ Le grandezze caratterizzanti le direzioni rispettive delle tangenti al meridiano geoidico, al parallelo geoidico ed alle due linee di curvatura ${ }^{5}$ ) della superficie, incrociantisi nel punto ogni volta considerato.

$2^{\circ}$ Il rapporto fra $i$ due raggi principali di curvatura della superficie.

Evidentemente la conoscenza di questi elementi, determinati in una fitta rete di punti, senza ricorrere ad alcuna speciale ipotesi, fornird un criterio di verifica della conclusione che abbia il Geoide forma poco diversa da quella di uno sferoide di rivoluzione, anzi potrebbe permettere di giungere, per una nuova via, a questa stessa illazione.

Le formule poi che vengono stabilite in questa prima parte della presente ricerca, conducono in modo assai semplice a stabilire l'equazione differenziale delle linee di curvatura (di una superficie qualunque), in coordinate geografiche.

5) Ometteremo naturalmente di ricordare qui le definizioni dei vari elementi matematici dei quali dovremo discorrere, trattandosi di cose universalmente note. 
Tutto quanto fu teste accennato forma argomento dei successivi $\mathrm{n}^{\mathrm{i}} 2$ e 3 della presente Nota.

Nei $\mathrm{n}^{\mathrm{i}} 4$ e 5 dimostro come si possa, in base agli stessi dati di osservazione inizialmente considerati, determinare il triedro fondamentale di una qualunque curva tracciata sulla superficie: ed a tale proposito considero in particolare il triedro fondamentale del meridiano geoidico. Cio perché l'esame di tale triedro presenta speciale interesse per la Geodesia. L'ulteriore studio delle formule che cosi si devono considerare conduce d'altro lato a stabilire l'equazione differenziale delle assintotiche (di una superficie), in coordinate geografiche.

$\mathrm{Nel} \mathrm{n}^{\circ} 6$ deduco, sempre dagli stessi dati di osservazioni, l'espressione dell'angolo di torsione di una geodetica del Geoide. Ed è opportuno soffermarsi su questo punto, in quanto l'elemento in parola ha, come è ben noto, importanza nello studio della deviazione di un arco elementare di una geodetica dall'arco di sezione normale (della superficie studiata), in uno degli estremi dell'arco di geodetica, e passante per l'altro estremo ${ }^{6}$ ).

Nel $n^{\circ} 7$ dimostro come, associando alle osservazioni gid indicate, la misura, per ciascun punto del Geoide, della lunghezza di un elemento di linea della superficie uscente da esso, si possano facilmente stabilire relazioni atte a determinare (in ciascuno dei punti in esame):

$\mathrm{I}^{\circ}$ I tre coefficienti della forma differenziale quadratica che rappresenta il quadrato dell'elemento lineare. del Geoide ( $I^{a}$ forma fondamentale) riferito ai meridiani e paralleli (geoidici) come linee coordinate.

$2^{\circ}$ I valori dei due raggi principali di curvatura.

L'avere determinati questi elementi, in un con le direzioni delle tangenti alle linee di curvatura, equivale, come è noto, ad aver determinati i tre coefficienti della $2^{a}$ forma fondamentale' della superficie.

Con cio si hanno tutti gli elementi necessari all'analisi intrinseca della superficie in esame.

Intenderemo poi sempre che, quando si parli, senz'altra designazione, di latitudine, di longitudine, di azimut, ci si riferisca rispettivamente a latitudine e longitudine astronomica, ad azimut astronomico.

Prima di procedere oltre è peró bene osservare che, come in tutte le ricerche geodetiche, qui pure si tratta di dedurre elementi della superficie matematica terrestre (Geoide) da risultati di osservazioni eseguite sopra la superficie fisica. Noi presupporremo quindi sempre senz'altro che i risultati di osservazioni, dei quali parliamo, siano già stati ridotti al livello del mare (o più propriamente al Geoide).

6) Le formule che generalmente si applicano, negli ordinari trattati di Geodesia, allo studio di tale deviazione, sono dedotte, presupponendo il Geoide coincidente con un ellissoide di rivoluzione: ma, cosa pure ben nota, le formule stesse si possono, in quell'ordine d'approssimazione nel quale sono comunemente condotti i calcoli geodetici, estendere anche al caso in cui si consideri una superficie qualunque, soggetta solo ad alcune condizioni generali della natura di quelle da noi prestabilite. 
E tale premessa è pienamente lecita, in quanto in primo luogo il prof. Pizzetri diede formule atte a ridurre al livello del mare le latitudini e le longitudini osservate ${ }^{7}$ ), senza porre alcuna speciale restrizione circa la forma del Geoide (passando per il tramite di una superficie di livello la quale passi per un punto maggiormente elevato del terreno nella regione che si studia). Tale riduzione richiede che alle osservazioni astronomico-geodetiche siano associate una livellazione di precisione e osservazioni gravimetriche eseguite in una fitta rete di pmit. Le formule del Pizzetri valgono per altro soltanto nell'ipotesi, del resto giustificata dall'esperienza, che siano da risguardarsi come quantità piccole del primo ordine i rapporti fra le elevazioni continentali e il raggio medio della Terra. E solo in tale ipotesi sono le predette formule di riduzione applicabili al caso nostro. Per quanto riflette la riduzione al Geoide degli azimut osservati, conviene premettere che $\mathrm{i}$ coseni direttori delle tangenti a linee della superficie in parola (Geoide) risulteranno, in ultima analisi, espressi in funzione di coordinate geografiche di punti. Perció le formule stesse, testè citate, del Pizzetri, oltre a fornire le correzioni da apportarsi agli angoli azimutali osservati per ridurli al livello del mare, forniranno pure le indicazioni occorrenti per l'uso dell'alt-azimut col quale tali angoli si misurano. Cid è troppo noto ed evidente a che sia -mestieri soffermarvisi più oltre.

In secondo luogo poi, intorno alla riduzione al livello del mare delle lunghezze misurate di archi di linee, della quale pure dovremo occuparci, è mestieri fare qualche considerazione che, allo scopo di non intralciare la trattazione vera e propria dell'argomento, rimandiamo alla fine del lavoro (al $\pi^{\circ} 8$ ).

2. La proposizione del prof. Pizzerri che occorre anzitutto assumere a fondamento è la seguente:

In ogni punto $P^{3}$ ) della superficie in esame sono rispettivamente tangenti coniugate della superficie stessa:

$I^{\circ}$ La tangente al meridiano geoidico e la linea cardinale Est.

$2^{\circ}$ La tangente al parallelo geoidico e la linea cardinale Nord.

Ora possiamo risguardare in ciascun punto $P$, come note, le linee cardinali Nord ed Est, in quanto la determinazione di tali direzioni costituisce un'operazione assai semplice e facile.

7) Przzettr, Sur la réduction des latitudes et des longitudes astronomiques au niveau de la mer [Astronomische Nachrichten, CXXXVIII (1895), colonne 353.358]. - Il PizzeTti stesso ritorno poi sull'argomento nella sua Nota: Intorno alla effettiva determinazione della superficie di livello terrestre entro regioni limitate [Rendiconti della R. Accademia dei Lincei, vol. IV (1.895), $2^{\circ}$ semestre, pp. 324-331] e nelle sue: Lezioni (litografate) sulla teoria meccanica della figura dei pianeti, tenute nella $\mathrm{R}$. Università di Pisa nell'anno rgor-902 $\left(\$ 3^{6}\right)$.

8) Come già si fece precedentemente, useremo sempre il simbolo $P$ a designare il generico punto della superficie (matematica) terrestre che ogni volta prenderemo in considerazione. 
Ora, quando della superficie in parola si consideri la rappresentazione sferica di Gauss ${ }^{9}$ ), segue dalle definizioni stesse di meridiano e parallelo geoidico che:

Le linee cardinali Nord ed Est, in ciascun punto (del Geoide), sono parallele la prima alla tangente all'imagine sferica ${ }^{\text {to }}$ ) del corrispondente meridiano geoidico, la seconda alla tangente all'imagine sferica del corrispondente parallelo geoidico:

Le proposizioni ora ricordate, associate all'altra pure ben nota che:

La tangente coniugata a quella di una curva di una superficie e la tangente all'imagine sferica della curva stessa sono perpendicolari fra di loro;

forniscono immediatamente questa regola semplicissima per determinare le direzioni delle tangenti rispettive al meridiano ed al parallelo geoidico del punto $P^{{ }^{\mathbf{x}}}$ ) del quale si presuppongono preventivamente individuate le coordinate geografiche: " $\mathrm{Si}$ considerino pertanto $i$ due punti del Geoide $P_{1}, P_{2}$, situati l'uno sulla (tangente alla) linea cardi. nale Est, l'altro sulla (tangente alla) linea cardinale Nord del punto $P$ ed entrambi a distanze da questo punto, le quali possano risguardarsi come infinitesime. Indi si determinino le coordinate geografiche di $P_{1}, P_{2}$ in guisa da individuare le direzioni delle normali $N_{1}, N_{2}$ al Geoide rispettivamente in detti punti. Si considerino i due piani $\pi_{1}, \pi_{2}$ l'uno individuato dalla normale al Geoide in $P$, che diremo brevemente $N$, e dalla parallela a $N_{1}$ che si imagini condotta per il punto stesso, l'altro individuato ancora da $N$ e dalla parallela a $N_{2}$, condotta pure per $P$. Evidentemente:

Le normali rispettive a $\pi_{1}, \pi_{2}$ condotte per $P$ saranno la prima la tangente al meridiano geoidico dello stesso $P$, la seconda la tangente all analogo parallelo geoidico.

Gli angoli formati (in $P$ ) da queste due tangenti con la linea cardinale Nord, vale a dire gli azimut del meridiano e del parallelo geoidico di $P$ nel punto stesso, azimut che diremo rispettivamente $\alpha, \beta$, si ottengono facilmente, espressi in funzione delle coordinate astronomiche dei tre punti considerati.

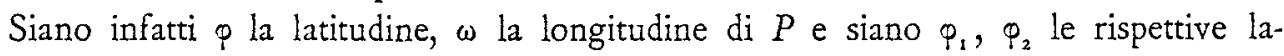
titudini, $\omega_{1}, \omega_{2}$ le longitudini di $P_{1}, P_{2}$. Poniamo brevemente:

$$
\tau_{\mathrm{r}}=\omega_{\mathrm{r}}-\omega, \quad \mathrm{J}_{2}=\omega_{2}-\omega \text {. }
$$

Diremo poi $X, Y, Z$ rispettivamente i tre coseni direttori della normale $N$ rapporto ai tre assi gid considerati $x, y$, z; designeremo con $X_{v}, Y_{v}, Z_{y}$ e con $X_{n}, Y_{n}, Z_{n}$ le analoghe terne di coseni direttori delle linee cardinali Nord ed Est del punto $P$. Naturalmente, per ciascuna di queste tre rette, supponiamo fissata la direzione da assumersi come positiva, secondo le convenzioni generalmente adottate ${ }^{\text {ra }}$ ). Avremo pertanto:

9) Reputo superfluo soffermarmi sulle citazioni bibliografiche relative ai concetti e teoremi di Geometria differenziale, ai quali qui dovró ricorrere, in quanto si tratterà sempre di cose notissime.

ro) Ogni qualvolta si parlerà di rappresentazione o di imagine sferica di una superficie, o di linee - punti di essa, si intenderà sempre di trattare della rappresentazione ottenuta appunto con la classica proiezione di Gauss.

II) In proposito v. Rerna, loc. cit. ${ }^{3}$ ).

I2) Vedi ad es.: Puccr, Fondamenti di Geodesia, vol. II, pp. 345-346. Del resto per le due linee cardin ali la direzione positiva risulta già fissata da quanto è detto nel $n^{\circ}$. 


$$
\left\{\begin{array}{lll}
X=\cos \varphi \cos \omega, & Y=\cos \varphi \operatorname{sen} \omega, & Z=\operatorname{sen} \varphi \\
X_{y}=-\operatorname{sen} \varphi \cos \omega, & Y_{y}=-\operatorname{sen} \varphi \operatorname{sen} \omega, & Z_{y}=\cos \varphi, \\
X_{n}=-\operatorname{sen} \omega, & Y_{n}=\cos \omega, & Z_{n}=0 .
\end{array}\right.
$$

I coseni direttori delle rette $N_{s}, \dot{N}_{2}$ riferite, ben si intende, agli stessi assi $x, y, z$ saranno rispettivamente:

$$
\begin{array}{lll}
\cos \varphi_{1} \cos \omega_{1}, & \cos \varphi_{1} \operatorname{sen} \omega_{1}, & \operatorname{sen} \varphi_{1}, \\
\cos \varphi_{2} \cos \omega_{2}, & \cos \varphi_{2} \operatorname{sen} \omega_{2}, & \operatorname{sen} \varphi_{12} .
\end{array}
$$

In base alla regola enunciata sarà allora agevole riconoscere che:

(2)

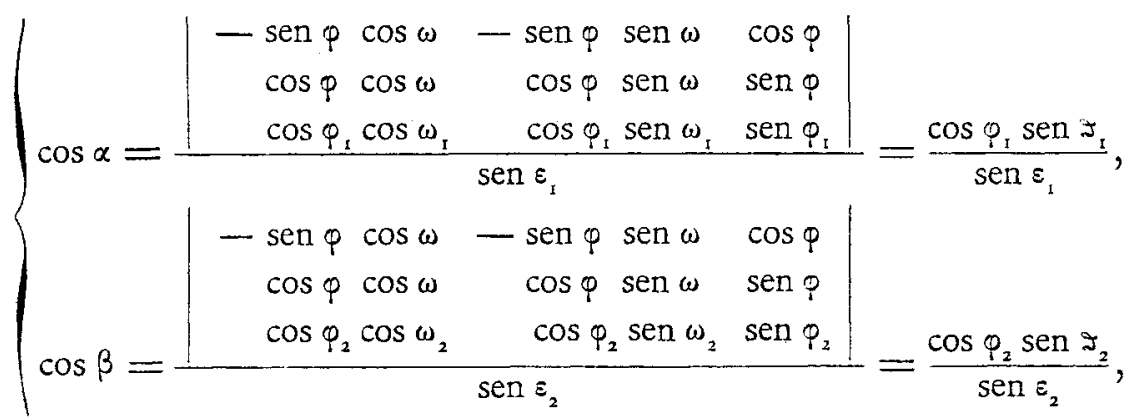

ove designino $\varepsilon_{r}, \varepsilon_{2}$ gli angoli della normale $N$ rispettivamente formati con le direzioni $N_{1}, N_{2}$. Saranno evidentemente $\varepsilon_{1}$, $\varepsilon_{2}$ dati dalle relazioni:

$$
\begin{aligned}
& \cos \varepsilon_{1}=\operatorname{sen} \varphi_{1} \operatorname{sen} \varphi+\cos \varphi_{1} \cos \varphi \cos \nabla_{1}, \\
& \cos \varepsilon_{2}=\operatorname{sen} \varphi_{2} \operatorname{sen} \varphi+\cos \varphi_{2} \cos \varphi \cos \nabla_{2} .
\end{aligned}
$$

Sarà ora opportuno scrivere l'espressione del coseno dell'angolo:

$$
\gamma=\alpha-\beta
$$

formato dalle tangenti del meridiano e del parallelo geoidico di $P$ nel punto stesso, espressione che facilmente si deduce dalle (2): e ciò facciamo perchè questa espressione dovremo considerare più innanzi.

Avremo pertanto:

$$
\left.\cos \gamma=\frac{\cos \varphi_{1} \cos \phi_{12} \operatorname{sen} \tau_{1} \operatorname{sen} \vartheta_{2}+\sqrt{\left(\operatorname{sen}^{2} \varepsilon_{1}-\cos ^{2} \varphi_{1} \operatorname{sen}^{2} \Xi_{1}\right)\left(\operatorname{sen}^{2} \varepsilon_{2}-\cos ^{2} \phi_{2} \operatorname{sen}^{2} \tau_{2}\right)}}{\operatorname{sen} \varepsilon_{1} \operatorname{sen} \varepsilon_{2}}{ }^{3}\right) .
$$

3. Dopo aver determinati gli angoli $\alpha, \beta$ è facile determinare pure gli angoli della linea cardinale Nord (nel punto $P$ ), con le analoghe tangenti alle linee di curvatura del Geoide, incrociantisi nel punto in parola.

Si dicano pertanto $R_{\mathrm{t}}, R_{2}$ i due raggi principali di curvatura del Geoide in $P$ : e sia $\chi$ l'angolo che una delle tangenti alle linee di curvatura (assi dell'indicatrice di DupIN

${ }^{13}$ ) In tutte le formule che servono a rappresentare funzioni trigonometriche di angoli, presupporremo sempre fissato, mercè opportune convenzioni, il verso da assumersi come positivo per le direzioni a cui si riferiscono gli angoli stessi. Cosi saremo dispensati dalla necessita di porre in tali formule il doppio segno, senza che per questo vi sia pericolo di dar luogo ad equivoci. 
relativa al punto stesso) forma con la analoga linea cardinale Nord. Allo scopo di fissare le idee supponiamo che la linea di curvatura considerata sia quella a cui compete il raggio di curvatura $R_{1}$. Allora, in virtù di una notissima relazione che lega $R_{1}, R_{2}$ agli angoli che due direzioni coniugate (nel punto $P$ ) formano con uno qualunque degli assi dell'analoga indicatrice di Dupin, avremo, ricordando pure il teorema del prof. PizzETTI citato nel $\mathrm{n}^{\circ}{ }^{I^{\mathbf{1}}}{ }^{\text {) }}$ :

$$
\left\{\begin{aligned}
\operatorname{tg} \chi \operatorname{tg}(\chi+\beta) & =-\frac{R_{2}}{R_{\mathrm{r}}}, \\
\operatorname{tg}\left(\chi+90^{\circ}\right) \operatorname{tg}(\chi+\alpha) & =-\frac{R_{2}}{R_{\mathrm{I}}} .
\end{aligned}\right.
$$

$\mathrm{Da}$ queste relazioni si ha evidentemente:

$$
\operatorname{tg}^{2} \chi \frac{\operatorname{tg} \chi+\operatorname{tg} \beta}{I-\operatorname{tg} \chi \operatorname{tg} \beta}+\frac{\operatorname{tg} \chi+\operatorname{tg} \alpha}{I-\operatorname{tg} \chi \operatorname{tg} \alpha}=0 .
$$

Ponendo brevemente, allo scopo di trasformare opportunamente quest'ultima equazione,

$$
\alpha=\alpha^{\prime}+90^{\circ}
$$

potremo appunto all'equazione in esame dare la forma:

$$
\left(\operatorname{tg}^{2} \chi+1\right)\left[\operatorname{tg}^{2} \chi+\operatorname{tg} \chi\left(\operatorname{tg} \alpha^{\prime}+\operatorname{tg} \beta\right)-1\right]=0 .
$$

$\dot{E}$ agevole riconoscere che quest'equazione di $4^{\circ}$ grado (rispetto a $\operatorname{tg} \chi$ ) ha due sole radici reali. Queste sono le radici dell'equazione:

$$
\operatorname{tg}^{2} \chi+\operatorname{tg} \chi\left(\operatorname{tg} \alpha^{\prime}+\operatorname{tg} \beta\right)-I=0 .
$$

Esse corrispondono a due valori di $\chi$ che differiscono fra di loro di $90^{\circ}$, essendo quelli che individuano le direzioni degli assi dell'indicatrice di Dupin, ossia delle tangenti rispettive alle due linee di curvatura.

Noto cosi $\chi$ mediante la (5) abbiamo evidentemente dalle (4):

$$
\frac{R_{2}}{R_{\mathrm{r}}}= \pm \sqrt{-\operatorname{tg}(\chi+\beta) \operatorname{tg}(\chi+\alpha)}
$$

relazione questa che serve a rappresentare il rapporto dei due raggi principali di curvatura della superficie in parola ${ }^{\mathbf{} 5}$ ), in ciascun suo punto in funzione di elementi tutti noti, in seguito alle osservazioni eseguite.

14) Le due relazioni che seguono furono già considerate anche dal Pizzetri nel suo lavoro inizialmente citato ${ }^{2}$ ).

I5) Qualora dalle osservazioni di cui sopra, risultasse che a meno di quantità piccole dell'ordine di grandezza degli errori di osservazione fosse in taluni punti : $\alpha=\beta-90^{\circ}=\chi=0$, le (4), (6) relative a ciascuno di questi punti assumerebbero forma indeterminata. Per rappresentare allora il rapporto $\frac{R_{2}}{R_{\mathrm{I}}}$ relativo a ognuno di questi punti, basterebbe però considerare per ciascuno di questi una qualunque direzione uscente da esso, determinando l'azimut $A$ di questa e determinando pure, con la regola sopra ricordata, la direzione coniugata a questa, della quale diremo $A^{\prime}$ l'azimut. Allora il rapporto $\frac{R_{2}}{R_{1}}$, per il punto ogni volta considerato, sarà dato dalla corrispondente espressione: $-\operatorname{tg} A \operatorname{tg} A^{\prime}$. 
Nel caso speciale del Geoide possiamo poi al radicale che figura nel secondo membro della (6) attribuire senz'altro il segno + , in quanto devesi ormai ammettere, in base a considerazioni ben conosciute, essere la curvatura (totale) del Geoide sempre positiva.

$\mathrm{La}$ (6) esprime pure una proprieta generale che compete a tutti i doppi sistemi di linee di una superficie, ai quali, come avviene di quello dei meridiani e paralleli geoidici, corrisponde nella rappresentazione sferica un doppio sistema ortogonale. La relazione stessa puó anche essere impiegata a porre in evidenza la notissima proprietà delle linee di curvatura di una superficie (la quale, ben si intende, non sia una sfera), di costituire, cioè, l'unico sistema doppio ortogonale di linee della superficie stessa, il quale si mantenga tale nella rappresentazione sferica.

La proprieta, già considerata nel $\mathrm{n}^{\circ} \mathbf{2}$, delle due linee cardinali del punto $P$, di essere cioè parallele rispettivamente alle tangenti ${ }^{\mathbf{1 6}}$ ) alle imagini sferiche del meridiano e del parallelo geoidico, permette di stabilire facilmente l'equazione differenziale delle linee di curvatura.

Infatti, come pure è noto, altra proprietà caratteristica delle linee di curvatura si è quella che le loro direzioni non sono deviate, nella rappresentazione sferica.

D'altra parte, l'elemento lineare $d \sigma$ della rappresentazione sferica del. Geoide, riferito alle linee $\varphi=$ cost., $\omega=$ cost. (cioè alle linee inviluppate rispettivamente dalle parallele alle singole linee cardinali Nord ed Est) è dato, in virtù della prima terna delle (I), dalla relazione:

$$
d \sigma^{2}=d X^{2}+d Y^{2}+d Z^{2}=d \varphi^{2}+\cos ^{2} \varphi \cdot d \omega^{2} .
$$

Percio, in base a quanto precede, e ricordando una delle notissime formule elementari della teoria delle superficie, è agevole riconoscere dovere lungo una linea di curvatura [della quale si designi sempre con $y$ l'azimut ${ }^{17}$ ) variante in generale da punto a punto] essere costantemente verificata la relazione:

$$
d p \operatorname{sen} \chi-d \omega \cos \varphi \cos \chi=0 .
$$

Pertanto la (8) sard evidentemente da assumersi come: l'equazione differenziale delle linee di curvatura (della superficie studiata) in coordinate geografiche.

Dall'equazione stessa si deduce subito la dimostrazione del già noto:

TeOrema ${ }^{\mathrm{r}}$ ). - Condizione necessaria e sufficiente a che le linee di curvatura (della superficie in esame) siano linee di azimut costantemente $=0$ e rispettivamente $=90^{\circ}$, si $\dot{e}$ che con le linee stesse coincidano ordinatamente $i$ meridiani ed $i$ paralleli geoidici.

4. Proponiamoci ora di determinare l'espressione dell'angolo di contingenza di una qualunque linea del Geoide. Consideriamo pertanto una qualunque linea $l$ della superficie in parola, passante per il punto generico $P$.

\footnotetext{
r6) Si tratta evidentemente di tangenti nell'imagine sferica dello stesso punto $P$. Nord.

${ }^{17}$ ) Cioè l'angolo della tangente alla linea in un dato suo punto con la analoga linea cardinale

18) Vedi Porncaré, Sur les déviations de la verticale en Géodérie [Bulletin Astronomique, t. XVIII (1901), pag. I57 e seg.].
}

Rend. Circ. Matem. Palermo, t. XXV (1 ${ }^{\circ}$ sem. sgo8). - Stampato it 12 dicembre 1907. 
Detto $A$ l'azimut di $l$ in $P$ è chiaro che i coseni direttori $\Lambda_{P}, M_{P}, N_{P}$ della tangente alla linea stessa, riferita ai tre assi $x, y, z$ saranno rispettivamente:

$$
\left\{\begin{aligned}
\Lambda_{P} & =-(\cos A \operatorname{sen} \varphi \cos \omega+\operatorname{sen} A \operatorname{sen} \omega), \\
M_{P} & =-\cos A \operatorname{sen} \varphi \operatorname{sen} \omega+\operatorname{sen} A \cos \omega, \\
N_{P} & =\cos A \cos \varphi .
\end{aligned}\right.
$$

Consideriamo quindi un secondo punto $P^{\prime}$ sopra $l$, prossimo a $P$, tanto che sia la lunghezza $d s$ dell'arco della linea stessa, compreso fra $\mathrm{i}$ due punti, una quantità piccola del primo ordine e siano tali pure le differenze fra le corrispondenti coordinate geografiche dei due punti, come pure fra gli azimut della linea in essi. Detti pertanto rispettivamente $\left.{ }^{19}\right): \varphi+d \varphi, \omega+d \omega, A+d A$ gli elementi che per $P^{\prime}$ hanno il medesimo significato che hanno, per $P$, le grandezze $\varphi, \omega, A$, e, detti $\Lambda_{P^{\prime}}, M_{P^{\prime}}, N_{P^{\prime}}$ i coseni direttori della tangente a $l$ in $P^{\prime}$, avremo evidentemente a meno di infinitesimi di ordine superiore:

$$
\begin{aligned}
\Lambda_{P^{\prime}}-\Lambda_{P} & =d A(\operatorname{sen} A \operatorname{sen} \varphi \cos \omega-\cos A \operatorname{sen} \omega) \\
& \quad+d \omega(\operatorname{sen} \omega \operatorname{sen} \varphi \cos A-\operatorname{sen} A \cos \omega)-d \varphi \cos A \cos \varphi \cos \omega, \\
M_{P^{\prime}}-M_{P}=d A(\operatorname{sen} A \operatorname{sen} \varphi \operatorname{sen} \omega+\cos A \cos \omega) & \quad-d \omega(\cos \omega \operatorname{sen} \varphi \cos A+\operatorname{sen} A \operatorname{sen} \omega)-d \varphi \cos A \cos \varphi \operatorname{sen} \omega, \\
N_{P^{\prime}}-N_{P}= & -(d A \operatorname{sen} A \cos \varphi+d \varphi \operatorname{sen} \varphi \cos A) .
\end{aligned}
$$

Per conseguenza avremo per l'angolo di contingenza $\varepsilon$ manifestamente l'espressione seguente (a prescindere dal segno):

$$
\begin{aligned}
\varepsilon & =\sqrt{\left(\Lambda_{p^{\prime}}-\Lambda_{P}\right)^{2}+\left(M_{p^{\prime}}-M_{P}\right)^{2}+\left(N_{p^{\prime}}-N_{P}\right)^{2}} \\
& =\sqrt{(d A-d \omega \operatorname{sen} \varphi)^{2}+(d \varphi \cos A+d \omega \operatorname{sen} A \cos \varphi)^{2}} .
\end{aligned}
$$

Ora il Pizzetri $\left.{ }^{\circ}\right)$ ha dimostrato essere la curvatura geodetica della linea $l$ in $P$ data da:

$$
C_{\mathrm{g}}=\frac{d A}{d s}-\frac{d \omega}{d s} \operatorname{sen} \varphi_{\mathrm{s}} .
$$

$\mathrm{Da}$ quest'ultima formula associata alla (IO) segue allora immediatamente essere la curvatura normale $C_{N}$ della $l$ in $P$ data da:

$$
C_{N}=\cos A \frac{d \varphi}{d s}+\operatorname{sen} A \cos \varphi \frac{d \omega}{d s} \text {. }
$$

La (I2) ci permette evidentemente di affermare che:

$$
\cos A d \varphi+\operatorname{sen} A \cos \varphi d \omega=0
$$

\section{é l'equazione differenziale delle assintotiche in coordinate geografiche ${ }^{2 x}$ ).}

r9) Per semplicità di scritura, si usi sempre il simbolo $d$ per designare indifferentemente sia incrementi finiti, piccolissimi, sia differenziali. Ció del resto non puó arrecare equivoco.

so) Contribuzione allo studio, etc., 1. c. ${ }^{2}$ ).

21) L'equazione in parola si poteva, come è evidente, dedurre anche dall'altra proprietà caratteristica delle assintotiche, che, cioè, nella rappresentazione sferica esse o, più propriamente, le loro tangenti in ciascun punto della superficie, vengono deviate di $90^{\circ}$. 
Da quest'equazione segue subito il

Teorema. - Affinche $i$ meridiani geoidici siano assintotiche della superficie studiata, è necessario e sufficiente che essi siano linee di azimut costantemente $=90^{\circ}:$ lanaloga condizione (necessaria e sufficiente) per $i$ paralleli è che essi siano linee di azimut costantemente nullo.

Le considetazioni ora svolte valgono in generale, quando si siano introdotte alcune evidenti modificazioni formali nelle definizioni, per qualunque superficie. E appunto per questo fu da me ritenuto opportuno considerare anche le assintotiche, il cui studio non presenta invece alcun interesse per la Geodesia, a motivo della circostanza già accennata potersi ritenere avere il Geoide in ogni punto curvatura (totale) positiva, in conseguenza di che le sue assintotiche sono, come è ben noto, imaginarie. Riserbandomi pertanto di ritornare eventualmente in altra occasione sull'argomento accennato, mi limiterò qui a far notare come dal teorema testè enunciato, posto in relazione con la (II), segua che un meridiano di una superficie, il quale sia una assintotica della superficie stessa, dovendo, come tale, essere una linea di azimut costante, sarà perciò pure una geodetica. Per conseguenza, come è ben noto: « un tale meridiano dovrà essere una retta».

Segue di qui, ove si ponga mente al teorema di ENNEPER sulla torsione delle geodetiche, che:

Una superficie sulla quale una delle due famiglie di assintotiche sia costituita dai meridiani è necessariamente una (superficie) sviluppabile ${ }^{22}$ ).

5. Dalle (IO), (II), (I 2) segue subito, detto $\psi$ l'angolo formato dalla normale principale della linea $l$ in $P$ con la analoga normale alla superficie, quest'altra relazione:

$$
\operatorname{sen} \psi=\frac{d A-d \omega \operatorname{sen} \varphi}{\sqrt{(d A-d \omega \operatorname{sen} \varphi)^{2}+(\cos A d \varphi+\operatorname{sen} A \cos \varphi d \omega)^{2}}},
$$

con la relazione analoga per $\cos \psi$. Le formule stesse permettono poi facilmente di costruire le espressioni dei coseni direttori si della normale principale come della binormale alla linea $l$ in $P$, rispetto ad una qualunque terna di assi coordinati.

Sarebbe poi superfluo entrare in proposito in minuti dettagli, trattandosi di cose di per sè evidenti. Ci limiteremo pertanto a scrivere per disteso le espressioni dei coseni direttori della binormale e della normale principale del meridiano geoidico di $P$, nel punto stesso, riferite alla terna costituita dalle linee cardinali Nord ed Est e dalla normale $N$ al Geoide nel medesimo punto $P$. Ciò perchè tali elementi sono compresi fra quelli che vanno presi in considerazione, allorchè, scendendo a particolari deduzioni intorno alla forma del Geoide, se ne vogliano studiare le deviazioni dalla forma di una superficie di rivoluzione.

Ora, quando le formule testè considerate si debbano riferire ad un meridiano geoidico, basterà porre in esse: $d \omega=0$. Pertanto, mantenendo il simbolo $\alpha$ a designare esclusivamente azimut di meridiani geoidici, è chiaro che $\mathrm{i}$ coseni degli angoli che il

22) Quindi, cosa pure nota, anche l'altra famiglia di assintotiche di una tale superficie coinciderebbe con quella dei meridiani. 
piano normale nel punto $P$ al meridiano geoidico del punto stesso forma col piano del meridiano astronomico di $P$ e col piano che incontra questo ad angolo retto lungo $N$, saranno rispettivamente: - $\operatorname{sen} \alpha, \cos \alpha$. E quindi avremo, per gli accennati coseni direttori della normale principale, disposti nell'ordine stesso nel quale furono indicati gli assi di riferimento, le espressioni seguenti:

$$
-\frac{\operatorname{sen} \alpha d \alpha}{\sqrt{d \alpha^{2}+(\cos \alpha d \varphi)^{2}}}, \quad \frac{\cos \alpha d \alpha}{\sqrt{d \alpha^{2}+(\cos \alpha d \varphi)^{2}}}, \quad \frac{\cos \alpha d \varphi}{\sqrt{d \alpha^{2}+(\cos \alpha d \varphi)^{2}}} .
$$

Gli analoghi coseni direttori della binormale saranno allora dati da:

$$
-\frac{\operatorname{sen} \alpha \cos \alpha d \varphi}{\sqrt{d \alpha^{2}+(\cos \alpha d \varphi)^{2}}}, \quad \frac{\cos ^{2} \alpha d \varphi}{\sqrt{d \alpha^{2}+(\cos \alpha d \varphi)^{2}}}, \quad-\frac{d \alpha}{\sqrt{d \alpha^{2}+(\cos \alpha d \varphi)^{2}}} \text {. }
$$

Ciò si vede subito, osservando che il coseno ed il seno degli angoli che la binormale forma con la $N$ sono rispettivamente: $-\operatorname{sen} \psi, \cos \psi$.

Naturalmente, allo scopo di determinare i sei coseni ora indicati, sarà necessario assumere il suaccennato punto $P^{\prime}$ sopra il meridiano geoidico di $P$, determinando, ben si intende, le coordinate geografiche di entrambi i punti e l'azimut in essi del comune meridiano.

6. Supponiamo ora che la linea $l$, di cui al $\mathrm{n}^{\circ} 4$, sia una geodetica del Geoide e proponiamoci di determinare l'espressione del suo angolo di torsione $n$ nel punto $P$. Manterremo in tutto e per tutto le notazioni adottate nel $n^{\circ} 4$ con l'analogo significato. Osserviamo pertanto che, rispetto agli assi $x, y, z$, i coseni direttori $\lambda_{P}, \mu_{P}, \nu_{P}$ della binormale in $P$ alla geodetica considerata saranno dati ordinatamente da:

$$
\begin{aligned}
& \lambda_{P}=\operatorname{sen} A \operatorname{sen} \varphi \cos \omega-\cos A \operatorname{sen} \omega, \\
& \mu_{P}=\operatorname{sen} A \operatorname{sen} \varphi \operatorname{sen} \omega+\cos A \cos \omega, \\
& \nu_{P}=-\operatorname{sen} A \cos \varphi .
\end{aligned}
$$

Ora, essendo la linea $l$ una geodetica, dovrd, in virtù della (II) essere sodisfatta la relazione :

$$
d A=d \omega \cdot \operatorname{sen} \varphi
$$

la quale è l'equazione differenziale delle geodetiche, in coordinate geografiche, data da BesSEL ${ }^{23}$ ).

Detti ora $\lambda_{P^{\prime}}, \mu_{P^{\prime}}, \nu_{P^{\prime}} \mathrm{i}$ coseni direttori (rispetto a $x, y, z$ ) della binormale alla geodetica considerata nel punto $P^{\prime}$, avremo evidentemente, tenendo conto della (14) e prescindendo da infinitesimi di ordine superiore:

$$
\begin{aligned}
\lambda_{P^{\prime}}-\lambda_{P} & =d \omega\left(\operatorname{sen}^{2} \varphi \cos \omega \cos A-\cos \omega \cos A\right)+d \varphi \cos \varphi \cos \omega \operatorname{sen} A, \\
\mu_{P^{\prime}}-\mu_{P} & =d \omega\left(\operatorname{sen}^{2} \varphi \operatorname{sen} \omega \cos A-\operatorname{sen} \omega \cos A\right)+d \varphi \cos \varphi \operatorname{sen} \omega \operatorname{sen} A, \\
\nu_{P^{\prime}}-\nu_{P} & =-d \omega \operatorname{sen} \varphi \cos \varphi \cos A+d \varphi \operatorname{sen} \varphi \operatorname{sen} A .
\end{aligned}
$$

Allora la misura $n$ dell'angolo di torsione della $l$, nel punto $P$, sard evidentemente

23) Il prof. Pizzettr, nel suo lavoro più volte citato ${ }^{2}$ ), stabili egli pure la (I4), deducendola appunto dalla (II), servendosi cosi di un metodo diverso da quello adottato da BESSEL. 
data da:

(15) $n=\sqrt{\left(\lambda_{p^{\prime}}-\lambda_{P}\right)^{2}+\left(\mu_{p^{\prime}}-\mu_{P}\right)^{2}+\left(\nu_{p^{\prime}}-\nu_{p}\right)^{2}}=d \varphi \operatorname{sen} A-d \omega \cos A \cos \varphi$.

L'espressione trovata per $n$ ha dunque la forma stessa del primo membro della (8). Perció detta formula fornisce una nuova dimostrazione del noto teorema che:

Se una linea di curvatura di una superficie è pure geodetica, essa è piana.

Dalla (I5) segue pure, in virt' di un notissimo teorema di BonNET, che la torsione geodetica $\frac{\mathrm{I}}{\tau}$, in $P$, di una qualunque linea della superficie in esame, tangente in $P$ stesso alla geodetica $l$ sarà al pari della torsione analoga di quest'ultima (il cui elemento d'arco si designi sempre con $d s$ ), data in valore assoluto da:

$$
\frac{\mathrm{I}}{\tau}=\operatorname{sen} A \frac{d \varphi}{d s}-\cos A \cos \varphi \frac{d \omega}{d s} \text {. }
$$

7. Passiamo ora a stabilire le formule che servono a dedurre, dai risultati di misure locali, i coefficienti $E, F, G$ dell'espressione:

$$
d s^{2}=E d \varphi^{2}+2 F d \varphi d \omega+G d \omega^{2},
$$

del quadrato dell'elemento lineare $d s$ della superficie in esame, riferita ai meridiani ed ai paralleli geoidici come linee coordinate. Indi si ricaveranno dagli stessi risultati di misure le espressioni dei due raggi principali di curvatura.

Supponiamo pertanto di avere misurata la lunghezza di un arco elementare $P P_{3}$ di una linea geodetica ${ }^{24}$ ) uscente dal punto $P_{\mathrm{r}}$. E supponiamo pure di avere determinate anche la latitudine $\varphi_{3}$ e la longitudine $\omega_{3}$ del punto $P_{3}$ (oltre, ben si intende, le analoghe coordinate $\varphi$, $\omega$ di $P$ ). Mantenendo le notazioni già introdotte, anche per rappresentare incrementi elementari finiti, stabiliremo che i varì elementi i quali figurano nella (I6) si riferiscano all'arco $P P_{3}$. Cosi ne rappresenti $d s$ la lunghezza, a prescindere da quantita che possiamo risguardare come infinitesimi di ordine superiore, mentre sia :

$$
d \varphi=\varphi_{3}-\varphi, \quad d \omega=\omega_{3}-\omega .
$$

Pertanto nella (I6) rimangono incogniti soltanto $E, F, G$.

In ciò che segue, uniformandoci all'uso generale, porremo, per brevità di scrittura,

$$
\begin{gathered}
\sum f_{x}=f_{x}+f_{y}+f_{z}, \quad \sum f_{X}=f_{X}+f_{Y}+f_{Z}, \\
\sum \varphi_{x_{X}}=\varphi_{x_{X}}+\varphi_{y Y}+\varphi_{x Z},
\end{gathered}
$$

ove designino rispettivamente $f_{\tau}, \varphi_{\tau_{1}, \tau_{2}}$ espressioni che dipendano l'una dal simbolo $\tau$, l'altra dai simboli $\tau_{1}, \tau_{2}$ sia direttamente che indirettamente in modo affatto generale.

Ora dalla seconda delle proposizioni enunciate nel $\mathrm{n}^{\circ} 2$, ove in pari tempo teniamo presente la (7), si deduce facilmente, in base agli elementi della teoria delle superficie, che i coseni direttori rispettivamente delle linee cardinali Nord ed Est tapporto agli assi

24) La convenienza di eseguire la misura indicata sopra un arco di geodetica risulterà da considerazioni che saranno svolte fra breve. 
$x, y, z$ si possono anche rappresentare con:

$$
\begin{aligned}
& \frac{\partial X}{\partial \varphi}, \quad \frac{\partial Y}{\partial \varphi}, \quad \frac{\partial Z}{\partial \varphi}, \\
& \frac{\mathrm{I}}{\cos \varphi} \frac{\partial X}{\partial \omega}, \quad \frac{\mathrm{I}}{\cos \varphi} \frac{\partial Y}{\partial \omega}, \quad \frac{\mathrm{I}}{\cos \varphi} \frac{\partial Z}{\partial \omega} .
\end{aligned}
$$

[Ció del resto si puó dedurre anche direttamente dalle ( $\mathrm{I})$ ].

Cosi, essendo:

$$
\sum\left(\frac{\partial X}{\partial \varphi}\right)^{2}=\mathrm{I}, \quad \sum\left(\frac{\partial X}{\partial \omega}\right)^{2}=\cos ^{2} \varphi
$$

ove si considerino le espressioni dei coseni direttori delle tangenti rispettive, nel punto $P$, al meridiano e al parallelo geoidico incrociantisi in esso, si dedurranno facilmente, in base alle considerazioni del $n^{\circ} 2$, le altre relazioni:

$$
\left.\frac{D^{\prime}}{\sqrt{E}}=\cos \varphi \operatorname{sen} \alpha, \quad \frac{D^{\prime}}{\sqrt{\bar{G}}}=\cos \beta^{25}\right),
$$

quando si sia posto, per brevita:

Dalle (I8) avremo dunque:

$$
D^{\prime}=\sum \frac{\partial x}{\partial \varphi} \frac{\partial X}{\partial \omega}=\sum \frac{\partial x}{\partial \omega} \frac{\partial X}{\partial \varphi} .
$$

$$
\sqrt{\frac{G}{E}}=\frac{\cos \varphi \operatorname{sen} \alpha}{\cos \varphi}
$$

D'altra parte, come pure è noto dagli elementi della teoria delle superficie:

$$
\sqrt{E G} \cos (\alpha-\beta)=F \text {. }
$$

Dividendo la (I 9$)$ membro a membro per la relazione precedente avremo:

$$
E=\frac{F \cos \beta}{\cos \varphi \operatorname{sen} \alpha \cos (\alpha-\beta)} \text {. }
$$

Invece, moltiplicando le stesse relazioni fra di loro membro a membro, otterremo manifestamente:

$$
\left(20^{\prime}\right) \quad G=\frac{F \cos \varphi \operatorname{sen} \alpha}{\cos (\alpha-\beta) \cos \beta} \text {. }
$$

Ove nella (I6) sostituiamo a $E, G$, le loro espressioni fornite rispettivamente dalle (20), $\left(20^{\prime}\right)$, otteniamo dalla stessa (I6) la seguente espressione di $F$, in funzione di grandezze ormai tutte note:

(2I) $\quad F=\frac{\operatorname{sen} \alpha \cos (\alpha-\beta) \cos \beta \cos \varphi d s^{2}}{\cos ^{2} \beta d \varphi^{2}+2 \operatorname{sen} \alpha \cos (\alpha-\beta) d \varphi d \omega+\cos ^{2} \varphi \operatorname{sen}^{2} \alpha d \omega^{2}}$.

La $(2 \mathrm{I})$ associata alle $(20),\left(2 \mathrm{O}^{\prime}\right)$ permette evidentemente di esprimere anche $E, G$,

25) Supporremo gia prefissato il verso da assumersi come positivo e sui meridiani e sui paralleli geoidici, in guisa da poter omettere, per i radicali $\sqrt{E}, \sqrt{G}$, il doppio segno. 
in funzione dei risultati delle misure eseguite. E sarebbe ormai superfluo scrivere per disteso tali espressioni.

Per determinare $R_{1}, R_{2}$ si ricorderd anzitutto che, detto $d S$ un'elemento d'area della superficie terrestre, comprendente il punto $P$ e detto $d \Sigma$ l'elemento corrispondente nella rappresentazione sferica, si avrà :

$$
d S=\sqrt{E G-F^{2}} d \varphi d \omega, \quad d \Sigma=\cos \varphi d \varphi d \omega .
$$

Ciò è ben noto da formule elementari di Geometria diffetenziale.

Per conseguenza la curvatura totale $\frac{\mathrm{I}}{R_{\mathrm{I}} R_{2}}$ del Geoide in $P$ si potrd rappresentare con :

$$
\frac{\mathrm{I}}{R_{1} R_{2}}=\frac{\cos \varphi}{\sqrt{E G-F^{2}}}
$$

D'altra parte, in base ad un'altra formula elementare di Geometria differenziale,

$$
\operatorname{sen}(\alpha-\beta)=\frac{\sqrt{E G-F^{2}}}{\sqrt{E G}}
$$

Le ultime due relazioni, moltiplicate membro a membro l'una per l'altra, forniscono la relazione:

$$
\frac{\operatorname{sen}(\alpha-\beta)}{R_{1} R_{2}}=\frac{\cos \varphi}{\sqrt{E G}}
$$

Associando a quest'equazione le (I9), (2I) si ottiene immediatamente:

$$
R_{2} R_{2}=\frac{d s^{2} \operatorname{sen} \alpha \cos \beta \operatorname{sen}(\alpha-\beta)}{\left.\cos ^{2} \beta d \varphi^{2}+2 \operatorname{sen} \alpha \cos (\alpha-\beta) d \phi d \omega+\cos ^{2} \varphi \operatorname{sen}^{2} \alpha d \omega\right)^{2}} .
$$

Associando questa relazione alla (6) si ottengono senz'altro i raggi principali di curvatura espressi in funzione di elementi tutti noti ${ }^{26}$ ).

Riassumendo le considerazioni svolte, possiamo affermare che la determinazione dei coefficienti delle due forme differenziali fondamentali relative al Geoide, in ciascun suo punto $P$, richiede le seguenti operazioni:

a) Determinazione delle linee cardinali Nord e Est relative al punto $P$ ogni volta considerato.

b) Determinazione delle coordinate geografiche, oltre che di $P$, dei punti designati rispettivamente con $P_{1}, P_{2}, P_{3}$.

c) Misura della lunghezza dell'arco di geodetica $P P_{3}$.

A queste operazioni vanno, come già si è detto, associate quelle necessarie per la riduzione al Geoide dei risultati delle osservazioni eseguite sopra la superficie fisica, terrestre.

Le formule che furono qui stabilite sussistono manifestamente solo quando siano,

26) Il prof. REINA, nella Nota citata 3 ), riconduce invece la determinazione di $R_{1}, R_{2}$ alla risoluzione di un'equazione di $2^{\circ}$ grado. 
nei punti che si considerano:

$$
\alpha \geqslant 0, \quad \beta-90^{\circ} \geqslant 0
$$

Quando invece le osservazioni astronomiche di cui al $\mathrm{n}^{\circ} 2$ indicassero che $\mathrm{i}$ valori di $\alpha$, della differenza $\beta-90^{\circ}$ (e per conseguenza anche di $\chi$ ) non eccedono, nella generalita dei punti terrestri, $i$ limiti entro i quali si possono ritenere compresi gli errori di osservazione, i procedimenti indicati per la risoluzione del problema propostoci dovrebbero essere modificati, in quanto le relazioni stabilite assumerebbero forma indeterminata.

Nel caso particolare in discorso, $\mathrm{i}$ meridiani e i paralleli geoidici, oltre a coincidere con le linee di curvatura del Geoide, sarebbero, come si puó anche dedurre facilmente dalle formule dei $\mathrm{n}^{\mathrm{i}} 4$ e 5 , pure linee piane ${ }^{27}$ ).

La (I6) assume allora, come è noto, la forma:

dove:

$$
\begin{gathered}
d s^{2}=E d \varphi^{2}+G d \omega^{2}, \\
E=R_{1}^{2}, \quad G=R_{2}^{2} \cos ^{2} \varphi .
\end{gathered}
$$

Nell'ipotesi in esame converrà, oltre alle operazioni a cui gì si accennó, eseguire la misura dell'azimut $A$ del considerato elemento di geodetica $d s$ (nel punto $P$ ). Una volta determinato tale angolo le ben note relazioni:

$$
\cos A=R_{\mathrm{r}} \frac{d \varphi}{d s}, \quad \operatorname{sen} A=R_{2} \cos \varphi \frac{d \omega}{d s}
$$

conducono senz'altro alla completa risoluzione della questione studiata.

In qualunque caso poi la conoscenza dei due coefficienti $E, G$ della prima forma differenziale fondamentale relativa alla superficie in parola porta con sè che siano note anche le derivate parziali di $x, y, z$ rispetto a $\varphi$, $\omega$. Ciò perchè, con le osservazioni astronomiche sopra accennate, rimangono evidentemente determinati i coseni direttori delle tangenti rispettive al meridiano e al parallelo geoidico di ciascun punto.

Avremo pertanto in ciascun punto del Geoide, in base a formule note dagli elementi della Geometria differenziale associate alle (9):

$\frac{\partial x}{\partial \varphi}=-\sqrt{E}(\cos \alpha \operatorname{sen} \varphi \cos \omega+\operatorname{sen} \alpha \operatorname{sen} \omega), \quad \frac{\partial x}{\partial \omega}=-\sqrt{G}(\cos \beta \operatorname{sen} \varphi \cos \omega+\operatorname{sen} \beta \operatorname{sen} \omega)$, con le due coppie di relazioni analoghe per $y, z$. (Evidentemente queste relazioni valgono per qualunque caso, compreso quello in cui: $\alpha=\beta-90^{\circ}=0$ ).

Qui $x$, $\beta$ si presuppongono espressi in funzione di $\phi, \omega$ : e tale loro rappresentazione si deduce evidentemente, mediante interpolazione, dai loro valori determinati in una fitta rete di punti.

Pertanto le equazioni ora scritte permettono teoricamente di stabilire, mercè semplici

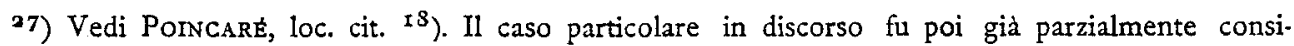
derato nella nota ${ }^{5}$ ). 
quadrature, l'equazione del Geoide in termini finiti ${ }^{28}$ ). Va pero notato che, in pratica, una tale determinazione si potrd fare soltanto entro limiti relativamente ristretti, in quanto, come già fu posto in luce dal BruHss ${ }^{29}$ ), il Geoide non è da risguardarsi come una superficie suscettibile di un'unica rappresentazione analitica, bensi come risultante dalla connessione di porzioni di superficie analitiche diverse. Circa poi ulteriori dettagli intorno ai limiti della applicabilita delle relazioni stabilite, non abbiamo che a riferirci alle considerazioni esposte in proposito dal prof. Rerna alla fine della sua Nota citata.

8. Ci rimane ora da indicare come si deduca la lunghezza dellarco $P P_{3}$ uscente dal generico punto $P$, dalle misure eseguite sopra la superficie fisica terrestre. In primo luogo tale arco dovrà essere sufficientemente limitato per potersene, con conveniente esattezza, rappresentare la lunghezza merce la (I6). Per meglio raggiungere tale intento e allo scopo pure di semplificare il lavoro materiale inerente alla misura e al tracciamento della linea considerata, convertà evidentemente eseguire la misura della distanza $P P_{\text {; }}$ lungo l'arco di sezione normale alla superficie terrestre in $P$, passante per $P_{3}$. L'elemento $P P_{3}$ potrà cosi, a motivo della sua limitata lunghezza, ritenersi confuso col corrispondente arco di geodetica.

La riduzione poi al livello del mare (o più propriamente al Geoide) di una distanza misurata sopra la superficie fisica terrestre richiede, come è ben noto, la conoscenza della grandezza del raggio di $\mathrm{I}^{\text {a }}$ curvatura della linea che si considera, in uno o piu punti del tratto misurato. E quando si tratta di basi geodetiche delle quali si determina la lunghezza, per procedere poi ad un'ordinaria triangolazione fondata su tale elemento, si attribuiscono a priori all'accennato raggio di curvatura valori corrispondenti a speciali ipotesi intorno alla forma del Geoide. Ma al contrario, nel caso nostro, la grandezza di questo raggio dipende appunto da quegli elementi tuttora incogniti che ci siamo proposti di determinare, valendoci dei soli risultati delle osservazioni. Non è peró difficile superare tale difficolta.

In primo luogo, date le nostre premesse, si potrà evidentemente all'arco $d s$, in ciò che concerne la deduzione della sua lunghezza dalle misure eseguite sul terreno, applicare la relazione ben nota che ordinariamente si impiega, a tale scopo, per le basi geodetiche.

Potremo cioè porre $\left.{ }^{30}\right)$ :

dove :

$$
d s=B^{\prime}-\sum \frac{b b}{R}-\frac{\mathrm{I}}{2} \sum b i^{2}
$$

28) Il Christoffel, nella Memoria citata ${ }^{x}$ ), ha ricondotta una siffatta determinazione all'integrazione di equazioni lineari a derivate parziali del $2^{\circ}$ ordine, le quali peró richiedono, per essere stabilite, la sola conoscenza dei raggi di curvatura principale $R_{\mathrm{I}}, R_{2}$. pag. $49, \$ 2]$.

29) Die Figur der Erde [Pubblication des Königl. Preussischen Geodätischen Institutes, .878 ,

30) Vedi ad es. Pizzetri, Trattato di Geodesia teoretica, Cap. VI. La relazione che segue sussiste, ben si intende, solo sotto la condizione, che noi supponiamo realizzata, che la misura in parola sia 
$I^{\circ} B^{\prime}$ rappresenti la lunghezza materialmente misurata del tratto della linea considerata, compreso fra $\mathrm{i}$ due punti della superficie fisica, terrestre, dei quali $P, P_{3}$ sono le proiezioni verticali sul Geoide.

$2^{\circ} \quad b$ rappresenti la lunghezza dell'apparecchio adoperato per la misura.

$3^{\circ} \mathrm{b}$ sia simbolo generale delle elevazioni sul Geoide degli estremi dei tratti di lunghezza = all'apparecchio di misura, nei quali si viene ad avere scomposta la linea segnata sul terreno. Le proiezioni verticali sul Geoide, di tali tratti, saranno elementi che nel loro insieme costituiscono la linea considerata $P P_{3}$.

$4^{\circ} R$ sia simbolo generale del raggio di $I^{2}$ curvatura di ciascuno dei predetti elementi, nei quali si puó concepire decomposto $P P_{3}$.

$5^{\circ} i$ sia simbolo generale dell'inclinazione all'orizzonte dell'apparecchio di misura nelle singole posizioni successivamente assunte neli'operazione in discorso. $E$ ciascun valore di $i$ si determina, in pratica, con twetodi ben noti.

Ora, attesa la relativa piccolezza di $B^{\prime}$ [che pure è il termine principale nel $2^{\circ}$ membro della (22)]. e della grandezza che, sia anche con grossolana approssimazione, si può attribuire a ciascun valore di $\frac{I}{R}$, mentre si puó ammettere che tale elemento varî con continuità, è lecito, come è noto, supporre, nel $2^{\circ}$ membro dell'equazione considerata, ciascun valore di $\frac{I}{R}=$ alla $I^{\text {a }}$ curvatura $\frac{\mathrm{I}}{\mathrm{P}}$ dell'arco $P P_{3}$ nel punto $P$. Cosi alla (22) potremo sostituire l'altra relazione:

$$
d s=B^{\prime}-\frac{1}{\rho} \sum b b-\frac{1}{2} \sum b i^{2} .
$$

Evidentemente possiamo, nelle ipotesi poste, porre con sufficiente esattezza:

$$
\frac{I}{p}=\frac{\varepsilon}{d s}
$$

detto $\varepsilon$ l'angolo delle due tangenti alla curva considerata, rispettivamente nei punti $P, P_{3}$. L'espressione dell'angolo in parola si dedurrá dai risultati di osservazioni astronomiche, con le formule del $\mathrm{n}^{\circ} 4$ : anzi, date le premesse fatte, si calcolerà mercè la (I2).

Dalle $\left(22^{\prime}\right),(23)$ potremo perció dedurre facilmente :

$$
\frac{I}{\rho}=\frac{\varepsilon}{B^{\prime}-\frac{I}{\rho} \sum b b-\frac{I}{2} \sum b i^{2}},
$$

dove tutto è noto, eccettuato $\rho$. Attesa la relativa piccolezza del secondo e del terzo

fatta sempre su un terreno di limitata elevazione sul livello del mare e pianeggiante. Allora si possono infatti ritenere come molto piccole le quantità che misurano sì i rapporti $\frac{b}{R}$ come le inclinazioni $i$ : cio, in base alle conclusioni che, almeno in via approssimata, le misure geodetiche sin qui fatte, permettono di trarre circa la grandezza di $R$. Del resto la restrizione accennata deve già essere ammessa, a che siano applicabili le formule precedentemente stabilite. 
termine nel denoninatore della rrazione al secondo membro di quest'equazione, riferiti a $B^{\prime}$, potremo, merce un evidente sviluppo di questa stessa frazione, limitato alla prima potenza di

$$
\frac{\mathrm{I}}{B^{\prime}}\left(\frac{\mathrm{I}}{i} \sum b b+\frac{\mathrm{I}}{2} \sum b i^{2}\right)
$$

dare a questa relazione l'altra forma:

$$
\text { (24) } \quad \frac{\mathrm{r}}{\rho}=\frac{\varepsilon}{B^{\prime}}\left(\mathrm{I}+\frac{\mathrm{I}}{\rho B^{\prime}} \sum b b+\frac{\sum b i^{2}}{2 B^{\prime}}\right) \text {. }
$$

$\mathrm{Da}$ quest'equazione segue subito potersi nel secondo membro della $\left(22^{\prime}\right)$, sostituire a $\frac{I}{\rho}$, senza errore apprezzabile, $\frac{\varepsilon}{B^{\prime}}$, ottenendo cosi, in definitiva, la seguente espressione di $d s$ :

$$
d s=B^{\prime}-\frac{\varepsilon}{B^{\prime}} \sum b b-\frac{\mathrm{I}}{2} \sum b i^{2} .
$$

Mantova, 4 novembre 1907 . 